\title{
ERRORES DE TRANSFERENCIA EN COMUNIDADES DE APRENDIZAJE EN LÍNEA POR APRENDIENTES DE ESPAÑOL COMO LENGUA EXTRANJERA (ELE) ${ }^{1}$
}

\section{TRANSFER ERRORS IN ONLINE LANGUAGE LEARNING COMMUNITIES BY LEARNERS OF SPANISH AS A FOREIGN LANGUAGE (SFL)}

\author{
Jessica Elejalde Gómez* \\ Anita Ferreira Cabrera**
}

\section{RESUMEN}

Las comunidades de aprendizaje de lenguas en línea ofrecen espacios gratuitos para practicar la escritura en la lengua meta, donde los aprendientes publican experiencias y/o relatos de diversos tipos. Esta escritura se gesta en el marco de una producción libre caracterizada por la naturalidad con las que son escritas, sin una intervención directa de la instrucción formal en una clase presencial. Este hecho podría reflejar un estado real de la lengua meta y, como consecuencia, evidenciarse la influencia de la lengua materna producto de la condición espontánea que ofrece la comunidad de aprendizaje en línea. En este contexto, se presentan los resultados de un estudio descriptivo basado en el análisis de errores de un corpus de 400 textos escritos por 80 aprendientes de ELE (Español como Lengua Extranjera) de diferentes comunidades. El objetivo es determinar cuáles son los errores de transferencia más frecuentes que ocurren en este tipo de ambiente de aprendizaje. La lengua materna de los sujetos es el inglés y el alemán con un nivel de proficiencia B1 declarado. Los resultados revelan que los errores de transferencia más frecuentes son: 1) los errores producto de interferencias de estructuras gramaticales como la omisión o adición de categorías gramaticales, 2) errores relacionados con la interferencia de estructuras y funciones sintácticas y 3) errores morfológicos y gráficos. Los resultados obtenidos en este estudio aportan una nueva perspectiva sobre el fenómeno de la transferencia según el ambiente de aprendizaje mediatizado por la tecnología y la producción escrita en ELE.

\footnotetext{
* Universidad de Concepción,Chile.jelejalde@udec.cl

** Universidad de Concepción,Chile. aferreir@udec.cl

1 Este artículo se circunscribe en la investigación en curso de la tesis doctoral de Jessica Elejalde Gómez bajo la dirección académica de la Dra. Anita Ferreira Cabrera, en el contexto del financiamiento de la Beca Conicyt PCHA Doctorado Nacional año 2013 Folio 21130823. El estudio presentado en este artículo se basa en el modelo metodológico y los procedimientos de análisis y procesamiento de corpus propuestos por la Dra. Anita Ferreira en su proyecto Fondecyt 1140651: "El feedback correctivo escrito directo e indirecto en la adquisición y aprendizaje del español como lengua extranjera". Universidad de Concepción, Concepción-Chile.
} 
Palabras claves: Comunidades de aprendizaje; errores de transferencia; corpus de aprendientes.

\begin{abstract}
Online language learning communities provide free of charge space to practice writing in the target language, where students can post their experience and/or various stories. This type of writing is created in an unconstrained context, characterized by the naturalness of how these texts are written, without direct intervention of formal in-class instruction. This could reflect a real state of the target language and, on account of this fact, the influence of the first language could be evidenced due to the spontaneous environment provided by an online learning community. In this context, this study presents the results of a descriptive study based on the error analysis from a corpus of 400 texts written by 80 Spanish learners from different communities. The objective is to determine what the most common transfer errors are in this type of learning environment. The subjects were native speakers of English and German who declared having a B1 level in Spanish. The results show that the most frequent transfer errors are: 1) errors due to interference of grammatical structure (e.g. omission or addition of grammar categories), 2) errors related to the interference of syntactic structures and functions, and 3) morphological and graphic errors. This research provides a new perspective on the transfer phenomenon in an environment mediatized by technology and written production in SFL.
\end{abstract}

Keywords: Learning communities; transfer errors; learner corpus.

\title{
INTRODUCCIÓN
}

Las comunidades de aprendizaje de Lenguas Extranjeras (LE)² en línea son espacios virtuales alojados en la red (RODRÍGUEZ, 2007), donde un grupo de personas que se encuentran en distintos lugares del mundo interactúan entre sî a través de un computador. Esto con el propósito de aprender de otras personas, realizar una práctica constante, intercambiar conocimiento o información sobre una LE. Allí, los miembros "comparten experiencias o intereses comunes y [...] se comunican entre sí para conseguir esos intereses" en la LE (MIRANDA, 2004, p. 7). De esta forma, la lengua objeto de estudio se aprende en un ambiente espontáneo donde la negociación del significado, la modificación del input, el output y la retroalimentación cobran un papel importante en el aprendizaje de la LE (LONG, 1996; LAMY Y HAMPEL, 2007).

Estas comunidades ofrecen posibilidades de un intercambio vía chat o por mensajes asíncronos entre pares de aprendientes y la publicación de escritos en la misma comunidad (cuaderno de notas) en la Lengua Objeto de estudio (LO). Con

2 El concepto de lengua extranjera refiere a que los aprendientes no están expuestos en su entorno inmediato al uso de la lengua objeto de estudio. 
respecto a la publicación, los aprendientes escriben en la LO experiencias y/o relatos de diverso tipo, los cuales son corregidos por un hablante nativo u otro aprendiente de la comunidad. Dichas publicaciones se gestan en el marco de una producción libre caracterizada por la naturalidad con la que son escritas, sin una intervención directa de la instrucción formal en una clase presencial. De esta forma, tienen una intención más comunicativa reflejando un estado real de la LO, y evidenciándose las estrategias a las que recurre un aprendiente cuando intenta comunicarse en la LO. Por consiguiente, considerar el análisis de este tipo de textos escritos permitirá observar nuevas tendencias de uso, describir procesos de la interlengua e identificar los errores de transferencia más frecuentes en este ambiente de aprendizaje apoyado por la tecnología.

En este contexto, se presentan los resultados de un estudio descriptivo con un enfoque mixto (cuantitativo-cualitativo) basado en el análisis de errores de un corpus de 400 textos escritos por 80 aprendientes de Español como Lengua Extranjera (ELE), producidos y publicados en diferentes comunidades de aprendizaje en línea. Los aprendientes tienen como lengua materna el inglés y el alemán con un nivel de proficiencia B1 declarado ${ }^{3}$. El objetivo principal es determinar y clasificar los errores de transferencia más frecuentes según la lengua materna en este tipo de textos acorde con el criterio etiológico.

El estudio se sustenta en investigaciones previas en el campo de Análisis de Errores (AE), específicamente en textos escritos producidos en ambientes de aprendizaje formal (aula de clases presencial), y se circunscribe en la interdisciplina de la Lingüística Aplicada, en el ámbito de la Adquisición de Segundas Lenguas (ASL) y la enseñanza de ELE (VÁZQUEZ, 1991; ALEXOPOULOU, 2006; QUIÑNONES, 2008; FERREIRA, ELEJALDE Y VINE, 2014). Los procedimientos metodológicos se sustentan en el modelo metodológico desarrollado e implementado por Ferreira (2014) en el proyecto FONDECYT No. 1140651.

Este artículo está organizado en las siguientes secciones: 2) se presentan los fundamentos teóricos del estudio, 3) se describe el estudio, la metodología y los procedimientos, 4) se exponen y discuten los resultados obtenidos en relación con la frecuencia de errores, las tendencias de uso erróneas y las principales problemáticas según la lengua materna de los aprendices, y 5) se presenta las conclusiones y proyecciones sobre los avances obtenidos en esta investigación.

3 Nivel declarado: refiere a la declaración que hace el aprendiente en su perfil sobre su nivel de dominio del español sin el respaldo de una prueba de nivel que lo certifique. En este caso, el nivel declarado en esta comunidad se determina según el Marco Común Europeo de Referencia (MCER, 2002). 


\section{FUNDAMENTOS TEÓRICOS}

Los recursos disponibles en la web se han convertido en una de las fuentes principales para la investigación en la Lingüística Aplicada, la Lingüística de Corpus (LC), la Adquisición de Segundas Lenguas (ASL) y los corpus de aprendientes de lenguas. Esto debido a la posibilidad de obtener datos auténticos del uso de la lengua real a gran escala disponibles en Internet. En este contexto, se presenta los fundamentos teóricos que sustentan el estudio en: las comunidades de aprendizaje en línea, el Análisis de Errores (AE) aplicados en ELE y los procesamientos de CLC (del inglés, Computer learner Corpora) para el análisis de córpora electrónicos.

\subsection{Las comunidades de aprendizaje de lenguas en línea}

Las comunidades "virtuales o en línea" ${ }^{4}$ surgen en la década de los 80 con el propósito de reforzar la práctica continua mediante el intercambio de conocimiento sobre el cual se tiene un interés común. El surgimiento de las tecnologías comunicativas y la posibilidad de la comunicación a distancia mediada por el computador han permitido que "un gran número de usuarios en un espacio común de intercambio de mensajes" (RODRÍGUEZ, 2007) interactuaran en torno a intereses comunes. Dichos intereses pueden estar destinados a la discusión, al intercambio de información o al aprendizaje de idiomas extranjeros.

Algunas comunidades de aprendizaje en línea como Italki, Polyglot Club y Livemocha $a^{5}$ son ejemplos documentados en cuanto a la disponibilidad de los recursos para la práctica y la comunicación en una lengua extranjera con distintos aprendientes alrededor del mundo. Allí se comparten historias coincidentes entre sus miembros y se ofrece oportunidades para interactuar en la LO. Esta práctica comunicativa se basa en la metodología del enfoque colaborativo donde los miembros de la comunidad trabajan en conjunto para conseguir un objetivo común (LAMY Y HAMPEL, 2007; RODRÍGUEZ, 2007).

Cabe destacar que estas comunidades, al estar disponibles en plataformas en línea mediatizadas por recursos computacionales, han hecho que estén basadas

\footnotetext{
4 Si bien existen diferentes denominaciones para este estudio se adopta la denominación "Comunidades de aprendizaje de lenguas en línea" (RODRÍGUEZ, 2007).

5 La comunicación en estas comunidades se realiza a través de chat, mensajes asincrónicos y publicación de textos en el contexto de la práctica de la LO, con el objetivo de esperar retroalimentación de otros miembros de la comunidad. Por lo general, los intercambios se concretan por medio de los intereses de cada miembro, los cuales están publicados en el perfil. Esto permite que aquellos miembros interesados en practicar unan LO, busquen a un compañero que quiera aprender la LM que dicho miembro posee y poder así generar una interacción real.
} 
casi exclusivamente en la escritura. En este aspecto, el registro continuo de las interacciones escritas (vivencias, relatos, etc.) reflejan una adaptación del valor simbólico de lo que representa una comunidad de aprendizaje (RODRÍGUEZ, 2007).

\subsection{La escritura en las comunidades virtuales y la influencia de la lengua materna}

Las comunidades de aprendizaje de lenguas en línea están basadas en la comunicación e intercambio de información mediada por el computador (del inglés, Computer-mediated Communication, CMC). La comunicación que surge allí se produce en la escritura, la lectura y oralidad vía Networked, donde se practica las habilidades lingüísticas para aprender una segunda lengua (WARSCHAUER, 2001). Entre estas posibilidades de uso, los aprendientes de las comunidades obtienen beneficios del intercambio con otros hablantes nativos, dado el interés por el aprendizaje de una lengua extranjera común o la del aprendiente (LAMY Y HAMPEL, 2007).

A través de la comunicación vía computador, estas comunidades de lenguas se han convertido en un espacio de interacción casi exclusivamente escrito, basado, principalmente, en el intercambio vía chat (sincrónico) o en la publicación de textos escritos resultados de la práctica en una lengua extranjera (asincrónico).

La escritura en estos sitios es libre, espontánea y el uso de la lengua meta es real en contextos de comunicación auténtica. De esta forma, se convierte en un proceso colaborativo propiciándose la interacción, la negociación del significado y la práctica de la lengua extranjera en diversas situaciones comunicativas (LONG, 1996; LAMY Y HAMPEL, 2007). En este aspecto, cuando el aprendiente se enfrenta al uso de la L2 en un ambiente natural, recurre a diferentes estrategias de comunicación o de aprendizaje, entre las cuales la lengua materna (LM) es la primera opción. El uso de la LM cobra importancia al suplir vacíos de información lingüística y otras estrategias como la sobregeneralización o neutralización permiten replicar reglas ya aprendidas en la L2.

Asimismo, los aprendientes en este ambiente de aprendizaje pueden controlar la ansiedad o el nerviosismo causados por la participación presencial en el aula de clases (LAMY Y HAMPEL, 2007). Por ello, la producción escrita digital propicia un nuevo procedimiento de escritura, donde su relevancia y análisis es similar a la de cualquier otro tipo de texto generado en otro medio físico (CASSANY, 2006). 


\subsection{La investigación en transferencia}

El fenómeno de la transferencia se describe como la influencia directa o indirecta de la lengua materna (LM) en la producción oral o escrita de un aprendiente de segundas lenguas (L2) de forma positiva o negativa. Esta influencia es un tipo de estrategia a la que recurre el aprendiente para solucionar obstáculos en situaciones comunicativas en las cuales hay una similitud entre la LM y L2 o para suplir un vacío de información lingüística de la L2. No obstante, la producción también puede verse afectada por otras condiciones externas como la transferencia de la instrucción, del ambiente de aprendizaje, de las estrategias de comunicación o de aprendizaje en la L2 (SELINKER, 1972).

Con respecto al ambiente de aprendizaje, los estudios enfocados en la transferencia presente en diferentes contextos, especialmente, los mediatizados por el computador y plataformas disponibles en internet son escasos. Gran parte de los estudios se ha realizado en el aula de clase con metodología de tipo experimental o descriptiva (MANCHÓN, 1985; ALEXOPOULOU, 2010, 2011) y enfocados a los errores producto de la interferencia negativa. En este aspecto, la visión de la producción escrita realizada a través de internet evidencia una escritura gestada en el marco de una actividad libre y espontánea, la cual podría verse influenciada por la LM u otras estrategias, al no ser una variable controlada en este tipo de ambiente.

La investigación en transferencia en clases presenciales ha arrojado estudios centrados en las estructuras lingüísticas que representan un área problemática como, por ejemplo, el uso de las categorías gramaticales (omisión y adición de elementos) dado que existen diferentes bifurcaciones por inexistencia o adición de estructuras gramaticales de la LM a la L2. Asimismo, la concurrencia fonológica de la LM influencia gran parte del sistema gráficoortográfico, observándose interferencias relacionadas con la pronunciación de la palabra en la L2 y la escritura de esta. A nivel sintáctico, funciones como la concordancia gramatical nominal y verbal presentan en menor grado (aun cuando se observa rasgos sintácticos en lenguas con estructuras similares a la L2) la influencia de los rasgos sintácticos de la LM sobre la L2, dado que la construcción de oraciones está determinada por el sistema lingüístico de la L2 diferente de la LM (VÁZQUEZ, 1991; ALEXOPOULOU, 2006; RAKASEDER Y SCHMIDHOFER, 2014). 


\subsection{El Análisis de Errores aplicado a la investigación en ELE}

El Análisis de Errores (AE) basa su fundamento en el reconocimiento del error como un indicio del proceso de aprendizaje en el cual se encuentra un aprendiente de lenguas (CORDER, 1975; LÓPEZ, 2009). Los errores muestran el conocimiento lingüístico de la lengua objeto de estudio que posee un estudiante de lenguas y forman parte del proceso natural del aprendizaje de una L2 (CORDER, 1975; ELLIS, 1997). El AE indica si estas desviaciones son producto de un estado inicial, del desarrollo o si se han fosilizado durante la conformación de la interlengua. Es así como el $\mathrm{AE}$ ha hecho parte de numerosas investigaciones que determinan no sólo la frecuencia de los errores, sino además su incidencia en el proceso de aprendizaje y adquisición de una segunda lengua (LÓPEZ, 2009).

Sin embargo, las críticas a este tipo de análisis se hicieron notar en las últimas décadas con el surgimiento de los enfoques comunicativos (LÓPEZ, 2009). Dado que el conocimiento lingüístico se centró más en la posibilidad de crear escenarios reales en contextos comunicativos, la importancia de la precisión gramatical y la adecuación contextual pasó a un segundo plano y, como consecuencia, los errores también. De igual forma, la obtención de las muestras ha sido ampliamente discutida debido a la naturaleza de estas, donde la elicitación de datos (observaciones en clase presencial) arrojaba resultados propios de un ambiente de aprendizaje influenciado por la instrucción y otras variables externas (LÓPEZ, 2009).

En el ámbito de ELE, la mayoría de estudios actuales sobre AE se ha desarrollado en ambientes de clases presenciales y enfocadas a la descripción de problemáticas en el campo de errores propios del sistema lingüístico. En este contexto es interesante mencionar los estudios de Vázquez (1991) llevado a cabo en clases de ELE a estudiantes de Alemania; el estudio de Quiñones (2008) con estudiantes de secundaria en Alemania, seguidos por Alexopoulou (2006) a estudiantes de pregrado de ELE en la Universidad de Atenas en Grecia, Rakaseder et al. (2014) en estudiantes universitarios de las carreras de gestión y asesorías de medios de una universidad de Austria y, Ferreira et al. (2014) en clases presenciales con estudiantes de intercambio de pregrado y posgrado de diferentes carreras en la Universidad de Concepción en Chile. Por consiguiente, la aplicación de un $\mathrm{AE}$ a los textos provenientes de publicaciones en comunidades de aprendizaje, arrojará nuevos indicios acerca de la interlengua en ELE y los errores producto de la influencia de la LM. 


\subsection{El análisis de errores y la investigación basada en corpus de aprendices}

La investigación en Análisis de Errores (AE) ha tomado una nueva dirección gracias a los avances tecnológicos e incorporación de procedimientos provenientes de la disciplina de la Lingüística de Corpus, específicamente, de la línea de investigación en corpus de aprendientes de lenguas (del inglés, Computer Learner Corpora). Esta evolución metodológica se ve reflejada en las técnicas para la recolección y tratamiento de textos, las cuales se apoyan en recursos tecnológicos para la recuperación y análisis de muestras a gran escala en formato digital (DAGNEAUX, DENNESS Y GRANGER, 1998; GRANGER, 2009; FERREIRA ET AL., 2014). Por medio de software especializado (programas para el etiquetado de errores) se identifica los errores, se procede a marcarlos con un sistema de anotación y se recupera la información para realizar un análisis de las tendencias de uso erróneo a partir de los datos cuantitativos arrojados por el software.

Asimismo, se puede determinar si estos errores se cometen de forma sistemática, con qué frecuencia ocurren y en qué contexto aparecen gracias a la posibilidad de obtener los extractos del texto donde está etiquetado el error (GRANGER, 2009). La metodología del AE apoyada por la tecnología (del inglés, Computer-aided-Error Analysis) procede siguiendo una serie de etapas propuestas inicialmente por Corder (1975). Estas etapas son: 1) la identificación de los errores, 2) la clasificación acorde con los criterios establecidos (estos pueden ser lingüísticos, pedagógicos, comunicativos), 3) la descripción de los errores acorde con una taxonomía de la modificación de la LO y 4) la explicación de la fuente donde se originan los errores.

\subsection{La clasificación y explicación de los errores}

La clasificación de errores se ha desarrollado acorde con diferentes visiones del error, sin embargo, los criterios lingüístico y etiológico han sido aplicados con mayor frecuencia en los estudios de AE (VÁZQUEZ, 1991; ALEXOPOULOU, 2006; FERREIRA ET AL., 2014). El criterio lingüístico describe los errores en un nivel categorial, morfológico, léxico, sintáctico, gráfico-ortográfico, y el etiológico explica el origen de los errores acorde con las estrategias comunicativas o de aprendizaje utilizadas por el aprendiente (interlinguísticas o intralinguísticas). Con respecto al etiológico, se ha dispuesto una serie de taxonomías donde se intenta explicar los procesos subyacentes a la 
influencia de la lengua materna y los procesos independientes de la L2 durante su conformación. Así en palabras de Alexopoulou (2006:23) "la explicación del error se refiere al proceso de adquisición del lenguaje e implica la interacción entre mecanismos internos y los factores externos que se ven involucrados". Es decir, se trata de dar cuenta de las estrategias de aprendizaje o comunicación a las que recurre el aprendiente, para inferir las causas que inducen al error a partir de los problemas identificados.

Estos criterios se fundamentan en la visión del error como un proceso sistemático que todo aprendiente de lenguas experimenta durante su aprendizaje de la L2 (ELLIS, 1997). Otros criterios propuestos, tales como el pedagógico y el comunicativo, se centran en el grado de comprensión, irritabilidad o perturbación que causa el error en diferentes situaciones comunicativas. El criterio pragmático y cultural se refiere a los errores de pertinencia propios del desconocimiento del uso contextual de la lengua meta (QUIÑNONES, 2009).

En lo que respecta a la descripción del error, la anotación de errores se basa en la identificación de las modificaciones y/o usos de la L2, la cual se puede clasificar en: 1) omisión de elementos oracionales o categorías gramaticales (OMI: "...veo *__ estatua de ${ }^{*}$ __ libertad..."), 2) falsa colocación (FC: "...las bonitas* casas...") de cualquier elemento dentro del sintagma nominal o verbal, 3) forma errónea de morfemas (FEM: la platocito*, cazabando*), 4) adición de elementos gramaticales (ADI: "...he decidido de* dejar..."), 5) falsa elección de la realización semántica de una palabra (FE: "...me he realizado* que no estaba bien...") y 6) falsa selección de cualquier categoría gramatical en vez de otra (FS: "...llegamos en* Madrid...") (QUIÑNONES, 2009; ALEXOPOLOU, 2006).

\section{METODOLOGÍA}

Esta investigación se sustenta en el modelo metodológico implementado en el proyecto de investigación Fondecyt No. 1140651 (FERREIRA, 2014). El objetivo principal de este estudio es determinar cuáles son los errores de transferencia más frecuentes acorde con el criterio etiológico en los textos escritos en comunidades de aprendizaje de lenguas en línea por aprendientes de ELE. Los resultados están clasificados según la lengua materna de los estudiantes con el objeto de observar las tendencias de uso de los errores en relación con el español. 


\subsection{Tipo de estudio}

El estudio que se presenta es de tipo descriptivo con un enfoque de análisis de datos mixto cuantitativo y cualitativo. El análisis cuantitativo se aborda desde los procedimientos de la investigación en corpus de aprendientes de lenguas para la obtención de las frecuencias y tendencias de uso. La interpretación cualitativa se determina a partir de los planteamientos del Análisis de Errores en el contexto de la investigación en ELE.

\subsection{Muestra}

La muestra es de tipo no probabilística intencionada, constituida por un total de 80 aprendientes de ELE de diferentes comunidades de aprendizaje en línea (Italki y Polyglot (lub). Las edades de los aprendientes fluctúan entre los 19 y los 30 años de edad. La lengua materna (LM) es el alemán (50\%) y el inglés (50\%). Los sujetos escribieron cinco textos cada uno distribuidos de la siguiente manera:

Tabla 1. Textos por sujeto y lengua materna

\begin{tabular}{|c|c|c|}
\hline Lengua Materna & No. Sujetos & Total textos \\
\hline Inglés & 40 & 200 \\
\hline Alemán & 40 & 200 \\
\hline Total & 80 & 400 \\
\hline
\end{tabular}

Con respecto a otras lenguas aprendidas después de la LM (alemán-inglés), los sujetos de L1 alemán tenían el inglés como L2 y en otros casos el francés como una L3 aparte del español. Los aprendientes cuya L1 era el inglés sólo tenían el español como L2 a excepción de un sujeto con una L2 francés.

\subsection{Procedimientos}

En función del objetivo general de este estudio, se construyó un corpus digital de aprendientes de ELE de textos escritos producidos en diferentes comunidades de aprendizaje en línea (Italki y polyglot club). Esto con la finalidad de obtener las frecuencias de errores y determinar las tendencias de uso erróneo por cada lengua materna. Las etapas para llevar a cabo los procesamientos se dividieron en: 1) recopilación del corpus, 2) identificación y clasificación de los errores y, 3) etiquetamiento de los errores en un software especializado. Los procedimientos 
se sustentan en el modelo metodológico para el procesamiento de corpus de aprendientes implementado y desarrollado por Ferreira (2014) y Ferreira et al. (2014). A continuación, se describe los procedimientos llevados a cabo durante el estudio.

\subsubsection{Recopilación del corpus}

La recopilación y procesamiento del corpus está basado en el modelo metodológico del proyecto Fondecyt No. 1140651 (FERREIRA, 2014) donde los criterios establecidos se sustentan en la línea de investigación de AE asistido por computador y corpus electrónico de aprendientes (TONO, 2003; FERREIRA ET AL., 2014) ${ }^{6}$. Esta recopilación se realizó de la siguiente forma:

a. Selección de los sujetos: se realizó de forma intencionada teniendo en cuenta la lengua materna inglés o alemán y el nivel de proficiencia declarado B1.

b. Recolección de textos: Selección y recolección de 5 textos por cada sujeto de forma intencionada considerando una extensión mínima de 100 y máxima de 200 palabras por texto.

c. Almacenamiento del corpus: Los textos fueron guardados en dos formatos, uno en word para el etiquetado manual y otro en $t x t$ para su posterior análisis en el software especializado Uam Corpus Tool ${ }^{7}$.

d. Revisión y depuración final: Revisión de los textos acorde con el contenido, con el objeto de identificar y mantener las secuencias textuales similares (narrativas y descriptivas), dado que la mayoría de los textos publicados relatan una experiencia, anécdota o historia personal.

\subsubsection{Identificación y clasificación de los errores}

Una vez terminada la construcción del corpus, se procedió a identificary señalar los errores de acuerdo con una taxonomía de clasificación previamente establecida y de forma manual en un archivo word. Luego, para tener una mayor precisión en

\footnotetext{
6 Las características corresponden a diferentes niveles tales como, tipo de lengua, modo de escritura, intención comunicativa, tipo de lengua, entre otros. Para profundizar dichas características consultar Ferreira, Elejalde y Vine (2014).

7 Software desarrollado en el contexto del Ministerio de Educación y Ciencia de España bajo el número de licencia HUM2005-01728/FILO (The WOSLAC Project).
} 
los errores identificados, se contó con la revisión externa de 6 traductores, 3 de alemán y 3 de inglés, de los cuales dos eran hablantes nativos de inglés y alemán residentes en Chile y cuatro hablantes nativos de español especialistas en inglés o alemán. Los traductores determinaron si los errores señalados con anterioridad podían explicarse a partir de la influencia de la LM o si obedecían a otro factor. Obsérvese un ejemplo de la identificación manual en word.

"...Él tenía un esquince en el tobillo y decidio a (Tarnsf-estruct-gramatical)

1 dejar la visita..." Sujeto 8, L1 inglés

Figura 1. Ejemplo identificación y clasificación de errores en word

Como se observa en el ejemplo, el error se marca en negrita y cursiva seguido por la etiqueta escrita entre paréntesis. La identificación del error trata de describir lo que el sujeto ha cometido y no la interpretación de lo que debería haber escrito o la corrección. En el caso de "decidio a" el sujeto adicionó una preposición producto de una transferencia de la LM. De este modo, la etiqueta que da cuenta de este error es la transferencia de una estructura gramatical que permite evidenciar la influencia de la LM en la adición de una categoría gramatical (preposición a). Al final del error, el traductor daba una explicación que diera cuenta del sistema lingüístico de la lengua materna y su influencia en el error cometido. Esta explicación se agregó como notas al pie en los archivos de word. Luego de esta revisión, se cotejó por cada error identificado la estructura y la explicación para determinar si efectivamente eran errores procedentes de la influencia de la LM o si eran parte del desarrollo de la interlengua del sujeto. Una vez identificados, se procedió a clasificarlos por problemática.

La taxonomía base fue el sistema de notación desarrollado en los proyectos de investigación Fondecyt No. 1110812 (Ferreira, 2011) y se depuró en el proyecto No. 1140651 por Ferreira (2014). Esta taxonomía incluye los criterios lingüístico y etiológico para determinar los errores de muestras elicitadas en ELE; sin embargo, para efectos de este estudio solo se consideró el criterio etiológico (FERREIRA, 2014) y la taxonomía para la descripción de la L2 dado el carácter del objetivo principal relacionado con los errores producto de la influencia de la LM. En este aspecto, se adecuó la clasificación de los errores haciéndose énfasis especial en los errores producto de la influencia negativa directa o indirecta de la lengua materna. Así la taxonomía modificada se basó en las dos categorías principales, las cuales se describe detalladamente. 
El criterio etiológico clasifica los errores en relación con la influencia de la lengua materna y las estrategias utilizadas por el aprendiz. Estos se dividen en dos categorías: 1) interlingual (transferencia y problemas relacionados con la L1), y 2) intralingual (problemáticas relacionadas con la independencia de la L2: sobregeneralización, neutralización, desconocimiento de la regla). Obsérvese la taxonomía aplicada en este estudio.

Cuadro 1. Criterio Etiológico basado en la taxonomía Fondecyt 1140651 (FERREIRA, 2014)

\begin{tabular}{|c|c|c|c|}
\hline Categoría & \multicolumn{2}{|c|}{ Tipo de error } & Descripción del error \\
\hline \multirow{11}{*}{ 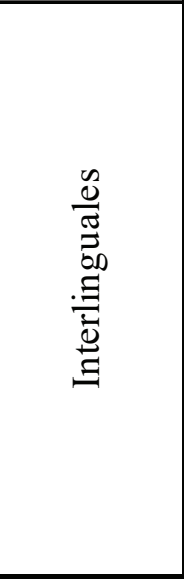 } & \multirow{6}{*}{ Transferencia } & Estruct-gramatical & \multirow{6}{*}{$\begin{array}{c}\text { Adición (ADI) } \\
\text { Omisión (OMI) } \\
\text { Falsa selección (FS) } \\
\text { Falsa colocación (FC) } \\
\text { Forma errónea (FE) }\end{array}$} \\
\hline & & Estruct-sintáctica & \\
\hline & & Léxica & \\
\hline & & Morfológica & \\
\hline & & Gráfica-ortográfica & \\
\hline & & Discursiva & \\
\hline & Traducción-lite & & \\
\hline & Cambio-de-cód & & \\
\hline & Interferencia-12 & & \\
\hline & Calco & & \\
\hline & Falso-cognado & & \\
\hline & Neutralización & & \\
\hline$\frac{\omega}{\Xi}$ & Sobregeneraliz & ación & \\
\hline $\overrightarrow{\sigma 0}$ & & Léxico creado por & rivación \\
\hline 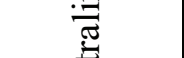 & Simnlificación & Aplicación incompl & de la regla \\
\hline$\Xi$ & 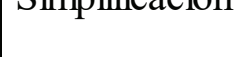 & Aplicación incorrec & de la regla \\
\hline & & $\overline{\text { Desconocimiento } \mathrm{d}}$ & a regla \\
\hline
\end{tabular}

Como se muestra en el cuadro 1 , los errores producto de la influencia directa o indirecta de la LM sobre la L2 se clasifican en 7 tipos de errores en la categoría interlingual: 1) transferencia, entendida como la influencia directa de la L1,2) traducción literal que induce a error cuando el sujeto traduce palabra por palabra una frase u oración, 3) cambio de código, referido al momento en el que el aprendiente escribe entre la producción de la L2 una palabra en su LM ("be viajado in europe* para tres meses"), 4) interferencia de una L2 ya sea a nivel léxico, gramatical o sintáctico, entendida como la influencia de otras lenguas aprendidas sobre la LO 
actual, 6) calco, cuando ocurre el traspaso de una unidad semántica a la L2 ("yo soy 22 años" estructura oracional para referirse a la edad, donde en español se realiza con el verbo tener) y, 7) falso cognado, relacionado con la interferencia negativa de la unidad semántica y gráfica del léxico en la LM a la L2.

Además, la subcategoría interlingual de transferencia se divide en seis tipos de errores: 1) Transferencia de estructura gramatical, en lo que refiere al uso de las categorías gramaticales ("yo conocí___ * mi amiga por internet" omisión de la preposición "a" para introducir complemento directo de persona donde en lenguas como el alemán o el inglés no se usa), 2) transferencia de estructura sintáctica, relacionada con la concordancia gramatical (género-número-atributo-predicado) en el sintagma nominal-verbal y el orden de los elementos en la oración, 3) transferencia léxica, referida al traspaso semántico y conceptual de la LM a la L2 ("gasté* toda mi vida en Boston" "pasé toda mi vida"), 4) transferencia morfológica de los rasgos de género y número, 5) transferencia gráfica-ortográfica, referida a la interferencia en la escritura (classico, necessito, elephante) y 6) transferencia discursiva relacionada con los conectores (naturalmente, de golpe). Por otra parte, la categoría intralingual se divide en errores producto de la neutralización entre la L1 y la L2, la sobregeneralización de las reglas de la L2 y la simplificación de la L2 (léxico creado por derivación, aplicación incompleta de la regla, aplicación incorrecta de la regla, desconocimiento de la regla).

\subsubsection{Etiquetamiento de los errores}

Una vez identificados y clasificados los errores acordes con los intereses de esta investigación, se procedió al etiquetamiento de estos en el software especializado Uam corpus tool. Este software tiene varias opciones, como el etiquetado automático, manual y semiautomático. Para efectos de este estudio se procedió al etiquetado manual, el cual permite sobre un sistema de notación identificar los errores para etiquetarlos en diferentes niveles. Esto permite etiquetar un error varias veces, como por ejemplo, en el sintagma "... *los canciones *classico..." (Sujeto 10, alemán texto No. 1), donde se evidencia dos errores, uno de tipo sintáctico (transferencia morfosintáctica) y otro de tipo gráfico (interferencia gráfica en la escritura de la palabra).

Terminado el proceso de etiquetamiento, se obtuvo una matriz general (todos los datos), otra matriz por lengua materna y una última matriz por sujeto con la frecuencia de los errores y los contextos del uso erróneo por cada categoría de error. Con estos datos se realizó el análisis cuantitativo (obtención de frecuencias, 
ocurrencias y concordancias) y cualitativo (explicación de las tendencias a partir de la LM), correspondiente para responder al objetivo general de este estudio.

\section{ANÁLISIS Y DISCUSIÓN DE LOS RESULTADOS}

Acorde con el objetivo principal, esto es, determinar cuáles son los errores de transferencia en ELE presentes en los textos escritos y publicados en comunidades de aprendizaje de lenguas en línea, se presentan los resultados de la obtención de frecuencias y ocurrencias del error acorde con el criterio etiológico.

Tabla No. 2 Resultados frecuencia de errores criterio etiológico

\begin{tabular}{|c|c|c|c|c|c|c|}
\hline Crite rio etiológico & Fr. Abs & $\%=\mathbf{2 3 8 3}$ & Ale mán & $\%=1071$ & Inglés & $\%=1312$ \\
\hline Interlingual & 1675 & $70 \%$ & 671 & $63 \%$ & 1004 & $77 \%$ \\
\hline Intralingual & 708 & $30 \%$ & 400 & $37 \%$ & 308 & $23 \%$ \\
\hline Total & 2383 & $100 \%$ & 1071 & $100 \%$ & 1312 & $100 \%$ \\
\hline Subtotal respecto del 100\% & \multicolumn{3}{|c|}{$45 \%$} & \multicolumn{2}{|c|}{$55 \%$} \\
\hline
\end{tabular}

La tabla 2 muestra un total de 2383 producciones erróneas distribuidas en las categorías interlinguales con un $70 \%$ (1675 errores) e intralinguales con un $30 \%$ (708 errores). Estos resultados de los porcentajes revelan que los fenómenos relacionados con la interferencia de la lengua materna en este tipo de textos son mucho mayores que los errores producto del desarrollo de la interlengua, es decir, los errores que se producen por la comprobación de hipótesis de las reglas y uso de la L2 (Ellis, 1997). De igual forma se evidencia que los aprendientes de L1 inglés son los que mayor frecuencia presentan con un 55\% del total de los 1312 errores a diferencia de la L1 alemán con un 45\% (1071 errores). 


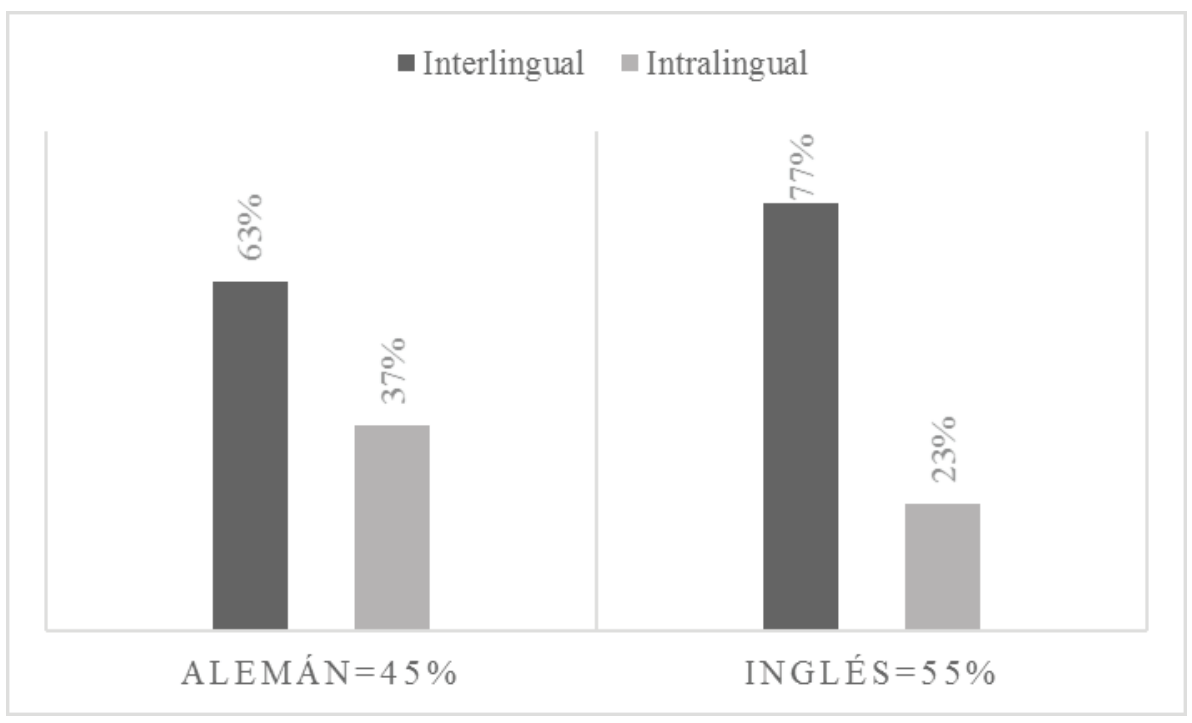

\section{Gráfico 1. Errores etiológicos por lengua materna}

Con respecto a los errores por lengua materna (gráfico 1), la LM inglés presenta un porcentaje mayor de errores interlinguales (77\%) a diferencia del alemán con un $63 \%$ respecto del total de cada categoría, esto es, 671 errores para el alemán y 1004 para el inglés. Por el contrario, los errores intralinguales en el alemán representan el $37 \%$, diferente del inglés con un $23 \%$. Esto puede interpretarse desde varias perspectivas teóricas, considerando que los sujetos de alemán para este estudio tenían otras lenguas aprendidas y el español como una de estas. En consecuencia, se podría sugerir que hay un menor compromiso cognitivo hacia la L1 propiciándose así la flexibilidad en la adquisición de otras lenguas como en el caso del alemán (MACWHINNEY, 2001).

Así en palabras de MacWhinney (2001) en su propuesta del modelo competitivo para ASL, una posible explicación corresponde a que la naturaleza interconectada de los procesos mentales promueve la transferencia de todo tipo de información, rasgos y características entre módulos emergentes y permeables. Cuando el sujeto adquiere la L1 en la infancia y se convierte en un aprendiente potencial de una L2, intenta aprender la L2 basándose en el sistema neurolingüístico de la L1. El sistema de la L2 no cuenta con una estructura lingüística y conceptual independiente de la L1 (denominado por MacWhinney como parasitismo) lo que obliga al aprendiente a depender de la L1 en muchas de las situaciones a las que tiene que enfrentarse en la L2. De este proceso se espera que el aprendiente 
construya representaciones de la L2 como un sistema independiente, a través del incremento de asociaciones directas entre las formas nuevas que adquiere de la L2 y sus representaciones conceptuales (MACWHINNEY, 2001). Por medio de la construcción directa de enlaces entre la pronunciación y sonidos en la L2, y de la reestructuración de los conceptos (nuevos o adquiridos en la L1), el aprendiente incrementa la automaticidad en el acceso independiente a la L2. Desde este punto de vista, los resultados señalan que los aprendientes cuya L1 es el alemán tienen un menor compromiso con la L1, dado que el español podría ser incluso una tercera lengua aprendida, a diferencia del inglés donde el español vendría a ser la segunda lengua.

\subsection{Resultados categoría interlingual}

Considerando que el objetivo principal de este estudio es determinar y clasificar los errores de transferencia acorde con la lengua materna en este tipo de ambiente de aprendizaje, se clasificaron los errores en las siguientes categorías ilustradas en la tabla a continuación.

Tabla 3. Resultados de errores categoría interlingual

\begin{tabular}{|c|c|c|c|c|c|c|c|}
\hline \multirow{9}{*}{ 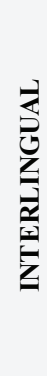 } & Categoría & Fr. Abs & $\%=1675$ & Ale mán & $\%=671$ & Inglés & $\%=1004$ \\
\hline & Transferencia & 1402 & $84 \%$ & 570 & $85 \%$ & 832 & $83 \%$ \\
\hline & Traducción-literal & 138 & $8 \%$ & 25 & $4 \%$ & 113 & $11 \%$ \\
\hline & Cambio-de-código & 39 & $2 \%$ & 9 & $1 \%$ & 30 & $3 \%$ \\
\hline & Interferencia-12 & 64 & $4 \%$ & 57 & $8 \%$ & 7 & $1 \%$ \\
\hline & Calco & 20 & $1,2 \%$ & 7 & $1 \%$ & 13 & $1,3 \%$ \\
\hline & Falso-cognado & 12 & $1 \%$ & 3 & $0 \%$ & 9 & $1 \%$ \\
\hline & $\begin{array}{r}\text { Total } \\
\end{array}$ & 1675 & $100 \%$ & 671 & $100 \%$ & 1004 & $100 \%$ \\
\hline & Subtotal & del $100^{\circ}$ & & & & & $\%$ \\
\hline
\end{tabular}

Como se muestra en la tabla 3, los errores producto de la influencia directa o indirecta de la L1 sobre la L2 se distribuyen con una frecuencia relativa de la siguiente manera: 1) transferencia con un $84 \%$, 2) traducción literal con un $8 \%, 3$ ) Cambio de código con un 2\%,4) interferencia L2 con un 4\%, 5) calco con un $1 \%$ y 6) falso cognado con un $1 \%$. De este tipo de errores interlinguales se observa que el inglés tiene una frecuencia relativa mayor, un $60 \%$ respecto del total, a diferencia del alemán con un $40 \%$. A continuación, se detalla la subcategoría de transferencia con el objeto de ejemplificar los errores identificados. 
Tabla 4. Resultados de errores de transferencia

\begin{tabular}{|c|c|c|c|c|c|c|c|}
\hline \multirow{10}{*}{ 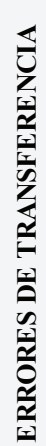 } & Categoría & Fr. Abs & $\%=1402$ & Ale mán & $\%=570$ & Inglés & $\%=832$ \\
\hline & Estruct-gramatical & 765 & $55 \%$ & 259 & $45 \%$ & 506 & $61 \%$ \\
\hline & Estruct-sintáctica & 279 & $20 \%$ & 135 & $24 \%$ & 144 & $17 \%$ \\
\hline & Léxica & 169 & $12 \%$ & 60 & $11 \%$ & 109 & $13 \%$ \\
\hline & Morfológica & 88 & $6 \%$ & 72 & $13 \%$ & 16 & $2 \%$ \\
\hline & Gráfica-ortográfica & 52 & $4 \%$ & 26 & $5 \%$ & 26 & $3 \%$ \\
\hline & Discursiva & 29 & $2 \%$ & 13 & $2 \%$ & 16 & $2 \%$ \\
\hline & Transf-inexistencia & 20 & $1 \%$ & 5 & $1 \%$ & 15 & $2 \%$ \\
\hline & \begin{tabular}{|c|} 
Total \\
\end{tabular} & 1402 & $100 \%$ & 570 & $100 \%$ & 832 & $100 \%$ \\
\hline & Subtotal & $\operatorname{del} 100^{\circ}$ & & & & & \\
\hline
\end{tabular}

Los resultados que muestra la tabla 4, sugieren que un 59\% de los errores corresponden al inglés, a diferencia del alemán con un $41 \%$ respecto del $100 \%$ (1402 errores). Si bien la diferencia no es sustantiva, puede observarse una mayor tendencia en el inglés con respecto a la transferencia de estructuras gramaticales (61\%) y sintácticas (17\%) y en el alemán un $45 \%$ de la transferencia gramatical y sintáctica con un 24\%. En relación con el estudio realizado por Ferreira et al. (2014), se evidencia tendencias similares donde los errores con mayor frecuencia son los del uso incorrecto de las categorías gramaticales. Esto corrobora que una de las áreas problemáticas es el uso correcto de las categorías gramaticales. En este sentido, se puede evidenciar que la selección de la forma esperada (target form) podría estar relacionada con la influencia directa o indirecta de la lengua materna.

Acorde con el objetivo de esta investigación, se observa que los resultados muestran una clara influencia de ambas lenguas en estudio (inglés y alemán) hacia el español, así como en otros análisis donde la escritura se ha obtenido en el contexto de clase (FERREIRA et al. 2014, FERREIRA Y LAFLEUR, 2015). La frecuencia de errores de transferencia refleja y sugiere en primera instancia que el ambiente de aprendizaje no afecta en mayor o menor cantidad la producción de estructuras provenientes de la LM. Esto podría interpretarse como una facilitación que el ambiente tecnológico propicia, favoreciendo al estudiante en su interacción de forma más espontánea y usando estrategias de comunicación que involucran la LM. Muchos de los casos observados en este estudio corresponden al desconocimiento de ciertas estructuras lingüísticas donde se observan errores como la omisión, donde el sujeto no identifica la regla del sistema o la desconoce. Esto conlleva al aprendiente de lenguas a usar su LM. Otros casos de transferencia pueden evidenciarse, como la falsa selección de estructuras gramaticales o sintácticas, donde el sujeto transfiere 
desde su LM dada la correspondencia que encuentra con la LO sin considerar si es adecuada la estructura para la situación comunicativa.

En este contexto, se detallan en siguiente apartado los errores más frecuentes en las categorías del uso erróneo, producto de la transferencia de estructura gramatical, sintáctica y morfológica.

\subsubsection{Transferencia de estructuras gramaticales}

En la subcategoría transferencia de estructura gramatical, entendida esta como la transferencia directa del uso de las categorías gramaticales de la LM hacia la L2 induciendo a error, se evidenció un total de 765 errores de los cuales 259 son productos de la influencia del alemán con un 34\%, y el inglés con 506 errores con un $66 \%$. Las tendencias que pueden identificarse (gráfico 2) son la omisión con 365 (48\%), la adición con 139 errores (18\%) y la falsa selección de categorías gramaticales con 261 errores (34\%). En consonancia con otros resultados, la tendencia del fenómeno de la transferencia se manifiesta principalmente en el uso de las categorías gramaticales, la cual representa un porcentaje mayor respecto de otras estrategias identificadas en las producciones erróneas (ALEXOPOULOU, 2006; VÁZQUEZ, 1991; FERREIRA ET AL., 2014; RAKASEDER ET AL., 2014). Obsérvese el siguiente gráfico.

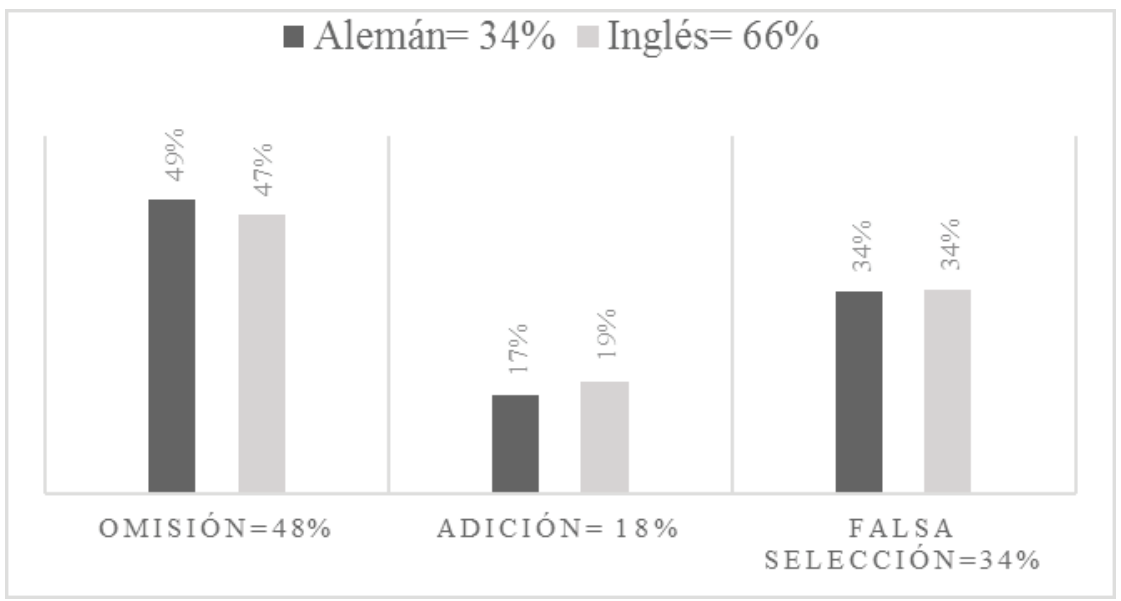

\section{Gráfico 2. Transferencia estructuras gramaticales}

En la tabla 5, se observa que los errores producto de la influencia del inglés representan un mayor porcentaje respecto del alemán, referido a la omisión de 
elementos gramaticales (47\%), seguidos de la falsa selección (34\%) y la adición (18\%) respecto del total de 506 errores en inglés y corresponden al 66\% de la totalidad de errores, esto es 765 .

Tabla 5. Errores transferencia estructura gramatical

\begin{tabular}{|c|c|c|c|c|c|c|c|}
\hline \multirow{5}{*}{ 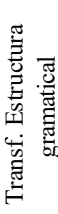 } & Tipo de error & Fr. Abs & $\%=765$ & Ale mán & $\%=259$ & Inglés & $\%=506$ \\
\hline & Omisión & 365 & $48 \%$ & 128 & $49 \%$ & 237 & $47 \%$ \\
\hline & Adición & 139 & $18 \%$ & 43 & $17 \%$ & 96 & $19 \%$ \\
\hline & Falsa selección & 261 & $34 \%$ & 88 & $34 \%$ & 173 & $34 \%$ \\
\hline & Total & 765 & $100 \%$ & 259 & $34 \%$ & 506 & $66 \%$ \\
\hline
\end{tabular}

Considerando que la omisión de elementos gramaticales es la categoría que mayor porcentaje de errores representa $(48 \%=365$ errores), en la tabla 7 se observa la distribución correspondiente a: 1) omisión de la preposición (180 errores), 2) omisión de artículos (110 errores), 3) omisión de pronombres (67 errores), y 4) otras omisiones referidas a categorías con uno o dos casos por sujeto (8 errores de omisión del verbo y del adverbio).

Tabla 6. Errores de omisión por transferencia de estructura gramatical

\begin{tabular}{|c|c|c|c|c|c|c|c|}
\hline \multirow{8}{*}{ 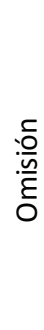 } & Tipo de error & Fr. Abs & $\%=365$ & Ale mán & $\%=128$ & Inglés & $\%=237$ \\
\hline & omi-preposición & 180 & $49 \%$ & 68 & $53 \%$ & 112 & $47 \%$ \\
\hline & omi-artículo & 110 & $30 \%$ & 33 & $26 \%$ & 77 & $32 \%$ \\
\hline & omi-pronombre & 67 & $18 \%$ & 21 & $16 \%$ & 46 & $19 \%$ \\
\hline & omi-verbo & 6 & $2 \%$ & 5 & $4 \%$ & 1 & $0 \%$ \\
\hline & omi-adverbio & 2 & $1 \%$ & 1 & $1 \%$ & 1 & $0 \%$ \\
\hline & omi-adjetivo & 0 & $0 \%$ & 0 & $0 \%$ & 0 & $0 \%$ \\
\hline & Total & 365 & $100 \%$ & 128 & $35 \%$ & 237 & $65 \%$ \\
\hline
\end{tabular}

Con respecto a los datos expuestos en la tabla 6, en comparación con el estudio de Ferreira et al. (2014) la omisión de la preposición se observa en 120 producciones erróneas, mientras que en este estudio corresponde a 180. Esto indica que el error de transferencia por omisión de la preposición es una problemática frecuente, debido a la variabilidad con la que se expresa y usa el sistema preposicional de cada lengua. Asimismo, se evidencia que los errores de este tipo también pueden ser explicados por la influencia directa o indirecta de la lengua materna más que de errores de tipo intralingual. Obsérvese algunos ejemplos que ilustran los errores etiquetados en esta categoría: 
- Omisión de la preposición

1) "...Quiero practicar mi español y ayudar * gente con inglés..." (Sujeto 10, L1 inglés)

2) "...Alemán es mi lengua materna y he comienzado * estudiar inglés en la escuela..." (Sujeto 14, L1 alemán).

3) "...Nos quedamos en un apartamento que estaba sólo * una cuadra de Times Square..." (Sujeto 11, L1 inglés).

El ejemplo 1 presenta una omisión de la preposición "a" en la introducción al complemento directo de persona. Esto puede deberse a una posible interferencia de la estructura gramatical del inglés donde no se utiliza la preposición para introducir este tipo de complemento. En la oración 2, la omisión de la preposición "a" en la perífrasis verbal, en español es necesario utilizar el nexo preposicional a diferencia del alemán donde en casos como este no se usa. En el ejemplo 3, se observa la tendencia de omitir la preposición "a" en estructuras que expresan la relación de distancia entre dos puntos, ya que en inglés se prescinde de esta. En español, esta relación se expresa con la estructura "estar $+\mathrm{a}^{\prime}$.

Estos resultados se relacionan directamente con otros estudios donde se identificó el uso erróneo de las preposiciones que presentan mayores problemáticas "a, de, por, para, en" producto de la influencia tanto del inglés como del alemán. Sin embargo, el estudio de las preposiciones debe clasificarse con criterios que den cuenta tanto de la heterogeneidad como del uso y las funciones dentro de la oración de cada preposición, así como lo plantean Perea (2007) y Ferreira y La Fleur (2015).

- Omisión del artículo

4) "...Quiero visitar la Estatua de *___Libertad..." (Sujeto 10, L1 alemán).

5) "...La participación [...] de las mujeres en *política ocurrieron (Sujeto 12, L1 inglés).

6) "...Italiano, * _ que he estudiado muy breve, también es útil por esto objetivo, pero he olvidado todos..." (Sujeto 14, L1 alemán).

Las oraciones 4 y 5 reflejan la tendencia en alemán e inglés de omitir el artículo en nombres de objetos en general o lugares en general. Por otra parte, en la oración 6 también se observa una omisión del artículo muy frecuente, relacionada con el uso del pronombre relativo "was" (que). Esto ocurre porque en alemán no se utiliza otro elemento adicional con el relativo que concuerde con el antecedente (sujeto) en este caso. 
Con este análisis, se podría sugerir en consonancia con los planteamientos de Bustos (1998), que la omisión de los elementos es una interferencia que proviene del desconocimiento de las reglas del sistema lingüístico de la LO, dándose lugar a la transferencia por inexistencia en el caso del uso de las categorías gramaticales (BUSTOS, 1998).

Teniendo en cuenta que la adición de categorías gramaticales es un tipo de error frecuente donde hay un traspaso directo de la L1 a la L2 en el uso de algunas categorías gramaticales, se evidenciaron 115 errores, un 34\% producto del alemán y un $66 \%$ del inglés.

Tabla 7. Errores de adición por transferencia de estructura gramatical

\begin{tabular}{|c|c|c|c|c|c|c|c|}
\hline \multirow{5}{*}{$\begin{array}{l}0 \\
: 0 \\
: 0 \\
0\end{array}$} & Tipo de e rror & Fr. Abs & $\%=115$ & Ale mán & $\%=39$ & Inglés & $\%=76$ \\
\hline & adi-preposición & 58 & $50 \%$ & 25 & $64 \%$ & 33 & $43 \%$ \\
\hline & adi-artículo & 25 & $22 \%$ & 8 & $21 \%$ & 17 & $22 \%$ \\
\hline & adi-pron & 32 & $28 \%$ & 6 & $15 \%$ & 26 & $34 \%$ \\
\hline & Total & 115 & $100 \%$ & 39 & $34 \%$ & 76 & $66 \%$ \\
\hline
\end{tabular}

Como se observa en la tabla 7, de estos errores cabe destacar la adición de preposiciones, artículos y pronombres con 115 errores distribuidos en el alemán (39 errores) e inglés (76 errores), siendo la preposición la que mayor frecuencia presenta. Obsérvese algunos ejemplos.

- Adición de la preposición

7) "...Nunca he visto una persona que no le gustó * de despertar muy temprano..." (Sujeto 11, L1 alemán)

8) "...Es porque decido *de tocar el piano..." (Sujeto 10, L1 alemán)

9) "...Él tenia un esquince en el tobillo y decidio *a dejar la visita guiada..." (Sujeto 8, L1 inglés)

En los ejemplos 7 y 8, la tendencia de adicionar la preposición "de" es muy frecuente en aprendientes de L1 alemán, ya que existen muchas construcciones

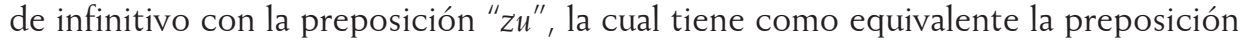
"de" o " $a$ ". En el ejemplo 9, se produce por medio de la traducción literal errónea un traspaso directo de la preposición " $a$ " en la perífrasis "*decidio a dejar la visita", (del inglés, "be decided to leave"). Esto ocurre debido a la adición equivalente de "to" con preposición " $a$ ". 


\subsubsection{Transferencia de estructuras sintácticas}

La categoría referida a la transferencia de estructuras sintácticas, se entiende como el traspaso directo de estructuras sintácticas (interferencia negativa) clasificadas en relación con el orden de las palabras y la concordancia gramatical. De un total de 279 errores distribuidos en el alemán con un 49\% (135 errores) y un 51\% (144 errores) en el inglés, puede observarse una mayor tendencia en la falsa selección de la concordancia gramatical de género o del número tanto en funciones sintácticas nominales como en el atributo en oraciones copulativas. Sin embargo, en el alemán se evidenciaron estructuras con elección errónea influidas por el género gramatical del sustantivo y por ende su concordancia con los adjetivos. Véase algunos ejemplos:

10) "...Las nuevas tecnologías son * más rápido, más fácil y barato..." (Sujeto 12, L1 alemán)

11) "...La costa pacífico es *muy caliente y búmedo..." (Sujeto 11, L1 inglés)

12) "...La *mejora escena para mi fue la primera..." (Sujeto 24, L1 alemán)

En alemán e inglés, al usar el adjetivo como atributo no concuerda en número y género con el sujeto porque son invariables (oraciones 10 y 11). En la oración 12, en alemán el adjetivo en función predicativa concuerda en género, número y caso gramatical con el sustantivo. El adjetivo "mejor" posee también una forma femenina que también concuerda con el sustantivo femenino "escena" (die beste Szene: la mejor escena). Estos hallazgos se condicen con los resultados de Rakaseder et al. (2014), donde los adjetivos en alemán solo son variables si se utilizan en el sintagma nominal en función predicativa.

En la concordancia entre sujeto y predicado también se observan algunas tendencias con el uso de sustantivos colectivos que en español están en singular, pero en alemán e inglés en plural.

13) "... Hay mas * gente que hablan español..." (Sujeto 14, L1 alemán)

14) ".. la gente *bailan y cantan con mucha alegría..." (Sujeto 3, L1 inglés)

En el ejemplo 13 "Leute", palabra alemana para "gente" es plural y el verbo se conjuga, por tanto en tercera persona del plural. El ejemplo 14 también muestra una influencia del inglés, puesto que el sustantivo "people" es plural; esta similitud entre ambas lenguas es propia de la familia a la que pertenecen: la germánica occidental. Otros casos referidos a la concordancia de género evidencian un número 
importante de transferencias en el uso del pronombre demostrativo neutro "esto" (del alemán, "Das ist") proveniente del alemán para introducir o presentar algo o a alguien independiente del género del objeto a introducir, así como se muestra en el siguiente ejemplo.

15) "... *Esto es mi primer intento de hacer un diario en Español..." (Sujeto 11, L1 alemán)

Considerando estos resultados, se sugiere que la tendencia de la falsa selección de la concordancia gramatical se corrobora con los hallazgos en el estudio de Pastor (2001) y Ferreira et al. (2014). Sin embargo, algunos patrones indican que hay transferencia de rasgos morfológicos del género producto de la influencia directa del alemán, que podrían interferir en la elección del género y por ende en la concordancia gramatical.

\subsubsection{Transferencia de rasgos morfológicos}

Con respecto a la transferencia de rasgos morfológicos, entendida esta como la interferencia negativa de los rasgos gramaticales de género, número o derivación de palabras de la L1 hacia la L2, es un tipo de error interesante dada la similitud en la asignación de la marca de género en los sustantivos. En esta categoría se identificaron 88 elecciones erróneas en total, donde se evidencia un número importante de errores (58 errores) producto de la influencia del alemán en la elección del género morfológico de los sustantivos. A diferencia del alemán, en el inglés el género y el número son invariables y dependen del contexto oracional. Véase algunos ejemplos.

16) "...En *otro parte del mundo son benéfico en las ciencias..." (Sujeto 12, L1 alemán)

17) "...Después tomé *una sauna..." (Sujeto 17, L1 alemán)

En la oración 16, el sustantivo "Teil" (parte) en alemán es masculino, por ello el sujeto transfiere el género eligiendo el determinante en masculino. La palabra "eine sauna" es femenino en el ejemplo 17. Otro hallazgo interesante y relacionado con los resultados de Rakaseder et al. (2014) es la tendencia de utilizar el masculino en sustantivos femeninos en español. 
18) "... No me gusta nada también presentar *los canciones..." (Sujeto 10, L1 alemán)

En la oración 18 "Canción" en alemán es "Lied" y tiene género neutro, más cercano al masculino. En palabras de Rakaseder et al. (2014:7) "los aprendientes muestran una clara preferencia por la forma masculina en detrimento de la femenina". Este tipo de errores muestra cómo incluso los rasgos de género, en el caso del alemán, pueden interferir negativamente, dada la similitud de las reglas en la marca de género propio de los sustantivos y su concordancia gramatical con los adjetivos.

\subsection{Resultados categoría intralingual}

Finalmente, los errores intralinguales corresponden a la aplicación independiente de reglas gramaticales de la L2, manifestándose principalmente en estrategias basadas en la simplificación y sobregeneralización del sistema lingüístico de la LO. Sin embargo, algunos de estos errores también poseen rasgos de la interferencia negativa de la LM hacia la L2, de los cuales puede destacarse la estrategia de la neutralización. Los errores producto de dicha estrategia corresponden a la interferencia por bifurcación tanto morfosintáctica como léxico semántica donde el sistema lingüístico de la L2 posee dos realizaciones, ya sean sintácticas o semánticas, de una sola en la LM del aprendiente. En este sentido, se presenta los errores de tipo intralingual con rasgos provenientes de la interferencia negativa de la lengua materna del aprendiz.

De un total de 708 errores un 56\% corresponde al alemán y un $44 \%$ al inglés respecto del $100 \%$. La neutralización, error que presenta mayor frecuencia, está asociada a la bifurcación entre las preposiciones (por y para), los verbos ser y estar, los adjetivos y adverbios (bien, bueno, mucho, muchas), los tiempos verbales del pretérito y la alternancia entre la elección del subjuntivo e indicativo. En ambas lenguas cada problemática sugiere una sola realización a diferencia del español que

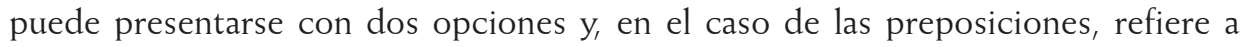
diferentes usos de corte contextual. Obsérvese algunos ejemplos:

- Neutralización de los verbos ser y estar

19) “... La protagonista se llama Katniss y tiene que * ser 10 dias con 22 otros jóvenes..." (Sujeto 10, L1 alemán).

20) “... Una cosa buena sobre Oxford es que *está muy pequeño [...] Londres *está mucho más grande..." (Sujeto 10, L1 inglés). 
La tendencia se observa en ambas lenguas, donde los verbos ser y estar sólo tiene una realización a diferencia del español. Para el aprendiente de ELE la distinción entre cualidades que clasifican el verbo ser (permanente o transitorio), la expresión de ubicación (estar) y el uso de los adverbios bien/mal induce al error, neutralizando finalmente la estructura a la más asimilada (RAKASEDER ET AL., 2014).

- Neutralización entre adjetivos y adverbios

21) “...Aunque el sistema del transporte pública es muy *bien, se parece que cada persona [...] quiere un carro..." (Sujeto 15, L1 inglés).

22) "...Casi todos los otros no se interesan que no estamos aprendiendo mucho, eso es *mal, creo..." (Sujeto 5, L1 alemán).

Esta neutralización es típica con elementos de la oración como los adverbios bien o mal confundidos con bueno y malo cuando van antecedidos por el adverbio muy.

- Neutralización de la preposición por y para

23) “...En las carteles puedes ver publicidad [... * * por productos como a la derecha de la compania Kodak..." (Sujeto 10, L1 alemán)

24) "... decidí aplicar *para escuela de postgrado en literatura de Inglaterra..." (Sujeto 1, L1 inglés)

En el ejemplo 23 del sujeto alemán, puede verse claramente la confusión entre las preposiciones por y para, donde en alemán podría ser posible solo con una realización "für". Lo mismo ocurre con el inglés (ejemplo 24), dado que tiene diferentes realizaciones y equivalencias con el español, como "for, to".

- Neutralización por alternancia subjuntivo e indicativo, y tiempos del pretérito

25) "... La fiesta organizamos el fin de semana, cuando *vamos a Cornwall (Suroeste de el país)..." (Sujeto 10, L1 inglés). Uso erróneo: tendencia de uso de alternancia entre el indicativo y el subjuntivo en estructuras cuando + futuro expresado en subjuntivo (en inglés, when + pres simple).

26) "... Cuando era jovén, yo tambien escribía en cuadernos, pero no *escribía seriamente hasta la escuela secundaria..." (Sujeto 1, inglés). Uso erróneo: falsa selección del pretérito imperfecto. Confusión por el uso debido a una sola realización en inglés (simple past). 
Los errores del verbo aquí expuestos reflejan una de las áreas que presenta mayor dificultad en el aprendizaje y adquisición del español como lengua extranjera, el uso del subjuntivo y la diferencia entre los usos de los tiempos verbales del pasado en el indicativo en ambas lenguas (RUIZ Y GUIJARRO, 2005; SÁNCHEZ, 2008; GUTIÉRREZ, 2004). En este sentido se corroboran las mismas tendencias observadas en estudios de la adquisición del pretérito en español por parte de aprendientes de alemán, donde la realización del tiempo pretérito simple y el imperfecto causa confusión en el momento de la escritura. Esto debido a que el pretérito perfecto compuesto es la forma lingüística predominante en el alemán (SÁNCHEZ, 2008; GUTIÉRREZ, 2004). Y con respecto al inglés, los estudios de errores a la luz de la lingüística contrastiva arrojan similitudes respecto de la neutralización del imperfecto y el simple para expresar el pasado en español debido a la distinción entre ambos pretéritos inexistente en el inglés (RUIZ Y GUIJARRO, 2005).

Considerando entonces los estudios realizados en Análisis de Errores, aplicados al aprendizaje y adquisición de ELE (VÁZQUEZ, 1991; ALEXOPOULOU, 2006; QUIÑNNES, 2008; FERREIRA ET AL. 2014), los resultados expuestos se corroboran con diferentes tendencias observadas en dichos estudios. Esto podría sugerir, sin ser concluyente, que los patrones de aprendizaje y adquisición del sistema lingüístico del español suelen ser similares tanto en contextos de aprendizaje presencial (instrucción formal) como en los ambientes apoyados por la tecnología como, por ejemplo, las comunidades de aprendizaje de lenguas en línea. No obstante, se observa en relación con las estrategias usadas por el aprendiz una mayor frecuencia de errores productos de la influencia de L1 (en este caso inglés y alemán) que en otros estudios percibidos en el aula han arrojado problemáticas relacionadas con las estrategias intralinguales, producto de la independencia de la L2 durante los estadios de aprendizaje intermedio avanzado (ALEXOPOULOU, 2006, QUIÑNONES, 2008).

\section{CONCLUSIONES}

El Análisis de Errores aplicado a la escritura de textos publicados libremente en comunidades de aprendizaje en línea permite describir el estado de la interlengua de los aprendientes en este contexto de aprendizaje. Dicha descripción aporta de manera significativa a los estudios realizados en el ámbito de la enseñanza, aprendizaje y adquisición del español como lengua extranjera (ELE), puesto que 
ejemplifica las tendencias de uso erróneo de ELE en interacciones escritas mediadas por el computador. Otra ventaja es la posibilidad de acceder a un número mayor de muestras disponibles en línea, a diferencia del proceso de instrucción donde se dificulta la recolección de muestras escritas.

Por consiguiente, los resultados obtenidos en este análisis de errores permiten sugerir, sin ser concluyente, que el tipo de ambiente tecnológico, si bien tiene mayores ventajas en cuanto a las posibilidades en el mejoramiento de la adquisición de la L2, evidencia al igual que otros contextos de aprendizaje errores producto de la lengua materna. Así, puede observarse que la interacción entre los miembros de la comunidad ocurre en un contexto enriquecido por la comunicación digital y la colaboración mutua entre los participantes, mejorándose el proceso de aprendizaje de la lengua en cuestión, pero no así aquellas interferencias inherentes a la producción en la L2 provenientes de la LM. Uno de los factores, que puede observarse del por qué esta interferencia ocurre, es la posibilidad que tiene cada miembro de escribir sin restricción, control o presión, lo que en muchas ocasiones podría generar interferencias directas o indirectas de la LM. En este sentido, el participante de esta comunidad es el único que ejerce control sobre su producción escrita y, por ende, sus estrategias tanto de comunicación como de aprendizaje pueden conducirlo a usar su lengua materna sin ser consciente de ello.

En la investigación sobre la transferencia, si bien existen estudios realizados en el aula de clase, la posibilidad de acceder a muestras donde el profesor o el curso de ELE no influencien los resultados, muestra errores que naturalmente se transfieren debido a que el estudiante debe resolver por sí solo las situaciones comunicativas a las que se enfrenta. El primer recurso al que acude es a su lengua materna, tratando de suplir vacíos de información lingüística que necesita aprender y asociar con la representación conceptual existente en la L1. El uso de la LM como estrategia de comunicación ha sido una de las más discutidas dada la factibilidad que tiene el hecho de que un aprendiz acuda a su LM cuando no posee el conocimiento lingüístico en determinada situación comunicativa. De igual forma, el control que tenga el estudiante sobre la producción de la LO dependerá de su conocimiento alcanzado lingüísticamente y su monitoreo constante para la autocorrección. No obstante, muchas estructuras del sistema lingüístico tienden a transferirse dada las características de cada lengua (distancia o cercanía entre las lenguas involucradas) y, en muchos casos, por el desconocimiento de la estructura usada en la LO.

Así los resultados muestran que, aun cuando se evidenció una mayor frecuencia de errores por parte de aprendientes cuya L1 es el inglés, el alemán también tuvo influencias importantes en aspectos gramaticales como la omisión y adición de 
elementos en la oración. Autores como MacWhinney (2001), argumentan que el proceso subyacente a la conformación de la L2 depende en la mayoría de casos del acceso que necesita el estudiante cada vez que debe incluir un nuevo concepto asociado a un nuevo sonido y su representación en la lengua materna.

Por otra parte, aunque la escritura en línea es espontánea y trae consigo una variedad de ventajas, no está exenta de la influencia de elementos externos que no pueden controlarse en este tipo de contexto. Es el caso del uso de los traductores en línea, los diccionarios, ayuda externa de un profesor, cuya influencia puede repercutir en el resultado del producto final, el cual se ha evaluado con exhaustividad. Así en palabras de Alexopoulou (2006), las limitaciones que se presentan en una investigación parten, principalmente, de la imposibilidad de estudiar todos los aspectos del desarrollo interlingüístico de los informantes y las condiciones en las que se obtiene los datos. De igual modo, cumplir con todas las condiciones de un ambiente libre de efectos en la mayoría de casos resulta incontrolable cuando se trata de análisis de aspectos tan dinámicos como la lengua. Y por último, los mismos informantes establecen limitaciones en los datos que proporcionan, ya que su veracidad, aun cuando se pueda corroborar, no está libre de influencias internas propias de su personalidad o aptitudes hacia el aprendizaje de la L2 (ALEXOPOULOU, 2006).

Otra de las limitaciones fue no contar con la posibilidad de evaluar el nivel real de los aprendices, quienes en su formulario de inscripción y en su ficha de perfil declaran un nivel de proficiencia sin un respaldo de un examen o certificado. Dicha limitación, si bien fue cautelada con la evaluación de los textos por dos expertos en el campo de la enseñanza y aprendizaje en ELE a través de una rúbrica con escalas basadas en las propuestas por DIALANG y el Marco Común Europeo de Referencia para las Lenguas (MCER, 2002), no deja de ser relevante contar con la certeza de conocer el nivel real de los estudiantes y poder realizar comparaciones con respecto al nivel.

Sin embargo, los resultados aquí expuestos trazan una posible descripción del proceso que ocurre en la interlengua de los aprendientes de ELE, cuya función permite corroborar no sólo tendencias observadas en estudios anteriores, sino que, a su vez, permite dar luces en cuanto a los errores que deberían ser tratados en la clase de ELE. Dichas tendencias pueden considerarse, además, para el diseño de propuestas para el tratamiento de errores de transferencia que contribuyan al mejoramiento en proceso de aprendizaje de una L2.

En este campo de investigación, los errores no dejan de ser una parte importante del proceso de aprendizaje de una L2, ya que, como lo afirma Corder 
(1975) nos revelan el estado en el que se encuentra el aprendiz, lo que es capaz y no de hacer y lo que debe replantearse para una futura intervención lingüística que mejore el aprendizaje de la L2.

Finalmente, para lograr un análisis que permita generalizar la muestra hacia una población determinada, se pretende en un futuro estudio, aumentar la muestra para poder establecer patrones en la adquisición del español. Esto debido a la posibilidad que entrega el acceso de muestras de escritura espontáneas, disponibles en este tipo de comunidades de aprendizaje en línea, donde el uso de la L2 es real y espontáneo. De igual manera, se espera incluir otras lenguas maternas para identificar tendencias, fenómenos y estrategias que usan los aprendientes de ELE a la hora de usar la lengua meta en este tipo de escritura espontánea.

\section{REFERENCIAS BIBLIOGRÁFICAS}

ALEXOPOULOU, A. (2006). Los criterios descriptivo y etiológico en la clasificación de los errores del hablante no nativo: Una nueva perspectiva. Porta Linguarum. v. 5, pp. 17-35.

ALEXOPOULOU, A. (2010). Errores intralinguales e interlinguales en la interlengua escrita de aprendientes griegos de e/le. Congreso de la Asociación Internacional de Hispanistas XVI: Nuevos caminos del hispanismo. Instituto de Cervantes. Madrid.

ALEXOPOULOU, A. (2011). El papel de la transferencia en los errores léxicos. Revista Nebrija de Linguiistica Aplicada a la Enseñanza de las Lenguas. v. 9, pp. 27-36.

BUSTOS, J. (1998). Análisis de errores: problemas de categorización. Cuaderno de filología Hispánica. $n^{\circ} 16$, pp. 11-40.

CASSANY, D. (2006). Análisis de una práctica letrada electrónica. Páginas de guarda: revista de lenguaje, edición y cultura escrita. $n^{\circ} 2$, pp. 99-112.

CORDER, S. P. (1975). Error analysis, interlanguage and second language acquisition. Language Teaching. v. 8, $\mathrm{n}^{\circ} 4$, pp. 201-218.

DAGNEAUX, E.; DENNESS, S.; GRANGER, S. (1998). Computer-aided error analysis. System. v. $26 \mathrm{n}^{\circ}$ 2, pp. 163-174.

DE EUROPA, C. (2002). Marco común europeo de referencia para las lenguas. Strasburgo: Consejo de Europa, Ministerio de Educación, Cultura y Deporte/Instituto Cervantes. ELLIS, R. (1997). SLA Research and Language Teaching. Nueva Yoork: Oxford University Press. FERREIRA, A (2011). Proyecto de investigación FONDECYT - CONICYT no.1110812: "Un Sistema Tutorial Inteligente para la Focalización en la Forma en la Enseñanza del Español como Lengua Extranjera". 
FERREIRA, A (2014). Proyecto de investigación FONDECYT - CONICYT no.1140651:"El feedback correctivo escrito directo e indirecto en la adquisición y aprendizaje del español como lengua extranjera" Universidad de Concepción, Chile 2014-2017.

FERREIRA, A.; ELEJALDE, J.; VINE, A. (2014). Análisis de Errores Asistido por Computador basado en un Corpus de Aprendientes de Español como Lengua Extranjera. Revista signos. v. 47, n 86, pp 385-411.

FERREIRA, A.; LAFLEUR, N. (2015). Analyse et description des erreurs prépositionnelles les plus fréquentes en espagnol L2. En Lingüística y Literatura. $n^{\circ}$ 68, pp. 57-79.

GRANGER, S. (2009). The contribution of learner corpora to second language acquisition and foreign language teaching. In: Aijmer, K., Corpora and language teaching. Philadelphia: Jhon Benjamins, v. 33, pp. 13-30.

GUTIÉRREZ, M. L. (2004). Problemas fundamentales de la gramática del español como 2/L. Madrid: Arcolibros.

LAMY, M. N.; HAMPEL, R. (2007). Online communication in language learning and teaching. Basingstoke: Palgrave Macmillan.

LONG, M. H. (1996). The role of the linguistic environment in second language acquisition.. In Ritchie, W. C.; Bhatia, T. K. (Eds.), Handbook of second language acquisition. Londres: Academic Press, pp. 413-468.

LÓPEZ, W. C. (2009). Error analysis in a learner corpus: what are the learners' strategies? En A survey of corpus-based research, p. 675-690. Disponible en: http://www. um.es/lacell/aelinco/contenido/pdf/45.pdf Acceso: 20 nov. 2014.

MACWHINNEY, B. (2001). The competition model: The input, the context, and the brain. Cognition and second language instruction, pp. 69-90.

MANCHÓN. M. (1985). Estudios de interlengua: análisis de errores, estrategias de aprendizaje y estrategias de comunicación. Revista española de lingüística aplicada. V. 1, pp. $55-75$.

MIRANDA, G. A. (2004). De los ambientes virtuales de aprendizaje a las comunidades de aprendizaje en línea. Revista digital universitaria. v.5, n 10, pp. 1-15.

PASTOR, S. (2001). La concordancia en la interlengua de los aprendientes de español como lengua extranjera. Estudios de Lingïística. v. 1, pp. 95-125.

PEREA, F. J. (2007). Análisis de errores y enseñanza de las preposiciones de la lengua española como lengua extranjera. MarcoEle revista de didáctica. v.5, pp. 1-13.

QUIÑNONES, A. V. (2008). Análisis de errores y de actuación en producciones escritas de aprendices alemanes de español. Estudio léxico-semántico. Tesis de doctorado en lingüística. Universidad Pablo de Olavide, Sevilla.

QUIÑNONES, A. V. (2009). El análisis de errores en el campo de ELE. Algunas cuestiones metodológicas. Revista Nebrija de Lingüística aplicada a la enseñanza de Lenguas. $n^{\circ} 5$, pp. $1-11$. 
RAKASEDER, J.; SCHMIDHOFER, A. (2014). Errores de producción de textos por parte de germanoparlantes en las primeras etapas de aprendizaje de ele. Marcoele revista de didáctica. $\mathrm{n}^{\circ} 19$, pp. 1-17.

RODRÍGUEZ, J. (2007). Comunidades virtuales, práctica y aprendizaje: elementos para una problemática. Revista electrónica Teoría de la Educación. v. 8, n³, pp. 6-22.

RUIZ, R.; GUIJARRO, J. R. (2005). Análisis contrastivo inglés-español: morfología, funcionalidad y semántica del imperfecto y del pretérito para estudiantes de español lengua extranjera. RedELE: Revista Electrónica de Didáctica ELE. n 4, pp. 1-16.

SÁNCHEZ, M. (2008). Dificultades de los hablantes nativos de alemán en el aprendizaje de los tiempos de pasado. RedELE: Revista Electrónica de Didáctica ELE. n 13, pp. 1-10.

SELINKER, L. (1972). Interlanguage. IRAL-International Review of Applied Linguistics. Language Teaching, v. 10, pp. 209-232.

VÁZQUEZ, G. E. (1991). Análisis de errores y aprendizaje de español/lengua extranjera. New York: Peterlang editorial.

TONO, Y. (2003). Learner corpora: Design, development and applications. In D. Archer, P. Rayson, A. Wilson \& T. McEnery (Eds.), Proceedings of the Corpus Linguistics 2003 Conference (pp. 800-809). Lancaster (UK): University Centre for Computer Corpus Research on Language.

WARSCHAUER, M. (2001). Online communication. The Cambridge guide to teaching English to speakers of other languages, pp. 207-212.

Recebido: 26/05/2016

Aceito: 15/10/2016 